Elect. Comm. in Probab. 3 (1998) 21-27

\title{
EVENTUAL INTERSECTION FOR SEQUENCES OF LÉVY PROCESSES
}

\author{
STEVEN N. EVANS AND YUVAL PERES \\ Department of Statistics \#3860, University of California at Berkeley \\ 367 Evans Hall, Berkeley CA 94720-3860, USA \\ e-mail: evans@stat.berkeley.edu peres@stat.berkeley.edu
}

Supported in part by NSF grants DMS-9703845 and DMS-9404391

submitted January 9, 1998; revised April 13, 1998

AMS 1991 Subject classification: 60J30, 60G17, 60G57, 60J45

Keywords and phrases: Lévy process, hitting probability, range, graph, random measure, random set, stationary

\begin{abstract}
Consider the events $\left\{F_{n} \cap \bigcup_{k=1}^{n-1} F_{k}=\emptyset\right\}, n \in \mathbb{N}$, where $\left(F_{n}\right)_{n=1}^{\infty}$ is an i.i.d. sequence of stationary random subsets of a compact group $\mathbb{G}$. A plausible conjecture is that these events will not occur infinitely often with positive probability if $\mathbb{P}\left\{F_{i} \cap F_{j} \neq \emptyset \mid F_{j}\right\}>0$ a.s. for $i \neq j$. We present a counterexample to show that this condition is not sufficient, and give one that is. The sufficient condition always holds when $F_{n}=\left\{X_{t}^{n}: 0 \leq t \leq T\right\}$ is the range of a Lévy process $X^{n}$ on the d-dimensional torus with uniformly distributed initial position and $\mathbb{P}\left\{\exists 0 \leq s, t \leq T: X_{s}^{i}=X_{t}^{j}\right\}>0$ for $i \neq j$. We also establish an analogous result for the sequence of graphs $\left\{\left(t, X_{t}^{n}\right): 0 \leq t \leq T\right\}$.
\end{abstract}

\section{INTRODUCTION}

Let $\mathbb{G}$ be a (not necessarily abelian) second countable, compact group. Consider an i.i.d. sequence $\left(F_{n}\right)_{n=1}^{\infty}$ of random closed subsets of $\mathbb{G}$. Suppose each $F_{n}$ is stationary in the sense that $x F_{n}$ has the same distribution as $F_{n}$ for all $x \in \mathbb{G}$. We are interested in conditions under which " $F_{n}$ doesn't keep slipping through the cracks left by $F_{1}, \ldots F_{n-1}$ ", by which we mean

$$
\mathbb{P}\left(F_{n} \cap \bigcup_{k=1}^{n-1} F_{k}=\emptyset \text { i.o. }\right)=0
$$

(observe that the probability of the event on the left-hand side is either 0 or 1 by the HewittSavage zero-one law). A necessary condition for (1) is that

$$
\mathbb{P}\left\{F_{i} \cap F_{j} \neq \emptyset \mid F_{j}\right\}>0 \text { a.s. for } i \neq j .
$$

In fact, (2) is equivalent to

$$
\lim _{n \rightarrow \infty} \mathbb{P}\left\{F_{n} \cap \bigcup_{k=1}^{n-1} F_{k}=\emptyset\right\}=0
$$


(see the beginning of the proof of Proposition 3.1), and it is tempting to conjecture that this condition is also sufficient for (1) to hold.

We present a counterexample to this conjecture in $\S 2$, and establish a valid sufficient condition for $(1)$ in $\S 3$.

The main application we have in mind is to the sample path properties of Lévy processes on the $d$-dimensional torus $\mathbb{T}^{d}$, where $\mathbb{T}$ is the circle of circumference $2 \pi$. Let $\left(Y^{n}\right)_{n=1}^{\infty}$ be an i.i.d. sequence of Lévy processes on $\mathbb{T}^{d}$ with $Y_{0}^{n}=0$. Write $\ell$ for the normalised Lebesgue measure on $\mathbb{T}^{d}$. Let $\left(U_{n}\right)_{n=1}^{\infty}$ be i.i.d. random variables on $\mathbb{T}^{d}$ independent of $\left(Y^{n}\right)_{n=1}^{\infty}$ and distributed according to $\ell$. Put $X_{t}^{n}=U_{n}+Y_{t}^{n}$. Recall that $Y^{n}$ is said to have resolvent densities if the measure $\int_{0}^{\infty} e^{-\alpha t} \mathbb{P}\left\{Y_{t}^{n} \in \cdot\right\} d t$ is absolutely continuous with respect to $\ell$ for each $\alpha>0$.

Theorem 1.1. Suppose that $Y^{n}$ has resolvent densities. The following two statements are equivalent.

(a) For all $T>0$,

$$
\mathbb{P}\left\{\exists 0 \leq s, t \leq T: X_{s}^{i}=X_{t}^{j}\right\}>0 \text { for } i \neq j
$$

(b) For all $T>0$,

$$
\mathbb{P}\left(\left\{\nexists 0 \leq s, t \leq T, 1 \leq k \leq n-1: X_{s}^{k}=X_{t}^{n}\right\} \text { i.o. }\right)=0 .
$$

We prove Theorem 1.1 in $\S 4$. Using similar ideas, we prove the following in $\S 5$.

Theorem 1.2. The following two statements are equivalent.

(a) For all $T>0$,

$$
\mathbb{P}\left\{\exists 0 \leq t \leq T: X_{t}^{i}=X_{t}^{j}\right\}>0 \text { for } i \neq j .
$$

(b) For all $T>0$,

$$
\mathbb{P}\left(\left\{\nexists 0 \leq t \leq T, 1 \leq k \leq n-1: X_{t}^{k}=X_{t}^{n}\right\} \text { i.o. }\right)=0 .
$$

In the final section, we describe a related unsolved problem concerning coalescing Lévy processes that motivated our interest in this topic.

\section{A COUNTEREXAMPLE}

The following counterexample shows that (2) is not sufficient for (1) to hold.

Take $\mathbb{G}=\mathbb{T}$. Take $E_{n}=\operatorname{cl}\left\{U_{n}+Z_{t}^{n}: t \in[0,1]\right\}$, where $\left(Z^{n}\right)_{n=1}^{\infty}$ is an i.i.d. sequence of $\alpha$-stable processes on $\mathbb{T}$ with $\frac{1}{2}<\alpha<1, Z_{0}^{n}=0$, and $\left(U_{n}\right)_{n=1}^{\infty}$ is an independent i.i.d. sequence of $\mathbb{T}-$ valued r.v. with common distribution $\ell$. Note that $E_{n} \cap\left[U_{n}, U_{n}+c\right]$ has Hausdorff dimension $\alpha$ almost surely for all $0<c<2 \pi$. Also, if $G$ is any fixed set with Hausdorff dimension greater than $1-\alpha$, then $\mathbb{P}\left\{\left(E_{n} \cap\left[U_{n}, U_{n}+c\right]\right) \cap G\right\}>0$ for all $0<c<2 \pi$. Therefore,

$$
\begin{aligned}
& \mathbb{P}\left\{\left(E_{i} \cap\left[U_{i}, U_{i}+c\right]\right) \cap\left(E_{j} \cap\left[U_{j}, U_{j}+d\right]\right) \neq \emptyset \mid E_{j}, U_{j}\right\}>0, \\
& \quad \text { a.s. for } i \neq j \text { and } 0<c, d<2 \pi .
\end{aligned}
$$

If $H$ is any closed, $\ell$-null subset of $\mathbb{T}$, then $\lim _{c \downarrow 0} \ell(\{x:[x, x+c] \cap H \neq \emptyset\})=0$ by bounded convergence. It is therefore possible to find a sequence $2 \pi>c_{1}>c_{2}>\ldots$ of positive constants 
such that $\sum_{n=1}^{\infty} \mathbb{P}\left\{\left[U_{n}, U_{n}+c_{n}\right] \cap \bigcup_{k=1}^{n-1} E_{k} \neq \emptyset\right\}<\infty$, and hence

$$
\mathbb{P}\left(\left[U_{n}, U_{n}+c_{n}\right] \cap \bigcup_{k=1}^{n-1} E_{k} \neq \emptyset \text { i.o. }\right)=0 .
$$

Let $\mu$ be a probability measure on $] 0,2 \pi\left[\right.$ such that $\left.\left.\sum_{n=1}^{\infty} \mu(] 0, c_{n}\right]\right)=\infty$, so that if $\left(V_{n}\right)_{n=1}^{\infty}$ are i.i.d. with common distribution $\mu$, then

$$
\mathbb{P}\left(V_{n} \leq c_{n} \text { i.o. }\right)=1 \text {. }
$$

Choose $\left(V_{n}\right)_{n=1}^{\infty}$ to be independent of $\left(\left(E_{n}, U_{n}\right)\right)_{n=1}^{\infty}$ and put $F_{n}=E_{n} \cap\left[U_{n}, U_{n}+V_{n}\right]$.

It is clear that $x+F_{n}$ has the same law as $F_{n}$ for all $x \in \mathbb{T}$. It follows from (3) that (2) holds. Now,

$$
\left\{F_{n} \cap \bigcup_{k=1}^{n-1} F_{k}=\emptyset\right\} \supseteq\left\{V_{n} \leq c_{n}\right\} \cap\left\{\left[U_{n}, U_{n}+c_{n}\right] \cap \bigcup_{k=1}^{n-1} E_{k}=\emptyset\right\},
$$

and it follows from (4) and (5) that the probability on the left-hand side of (1) is 1 in this case.

\section{A SUFFICIENT CONDITION}

Given two finite Borel measures $\mu$ and $\nu$ on $\mathbb{G}$ and $x \in \mathbb{G}$, define finite measures $\mu * \nu, \tilde{\mu}, \sigma_{x} \mu$, and $\tau_{x} \mu$ by $\mu * \nu(f)=\iint f(y z) \mu(d y) \nu(d z), \tilde{\mu}(f)=\int f\left(y^{-1}\right) \mu(d y), \sigma_{x} \mu(f)=\int f(x y) \mu(d y)$, and $\tau_{x} \mu(f)=\int f(y x) \mu(d y)$, respectively. As usual, write supp $\mu$ for the closed support of a finite Borel measure $\mu$. Recall that $\mathbb{G}$ is unimodular. That is, there is a unique Borel probability measure $\lambda$ (the Haar measure) such that $\sigma_{x} \lambda=\lambda$ for all $x \in \mathbb{G}$ and the measure $\lambda$ also has the property that $\tau_{x} \lambda=\lambda$ for all $x \in \mathbb{G}$.

Proposition 3.1. Let $\left(M_{n}\right)_{n=1}^{\infty}$ be an i.i.d. sequence of random probability measures on $\mathbb{G}$ such that $\sigma_{x} M_{n}$ has the same distribution as $M_{n}$ for all $x \in \mathbb{G}$ and $M_{i} * \tilde{M}_{j}$ is almost surely absolutely continuous with respect to $\lambda$ for $i \neq j$ with a density that is in $L^{2}(\lambda \otimes \mathbb{P})$. Then (1) holds for $F_{n}=\operatorname{supp} M_{n}$.

Proof. We want to show that $\mathbb{P}\left(A_{n}\right.$ i.o. $)=0$, where $A_{n}$ is the event that $F_{n}$ does not intersect $\bigcup_{k=1}^{n-1} F_{k}$. Observe that

$$
\begin{aligned}
\mathbb{P}\left(A_{n}\right) & =\mathbb{E}\left[\prod_{k=1}^{n-1} \mathbf{1}\left\{F_{k} \cap F_{n}=\emptyset\right\}\right] \\
& =\mathbb{E}\left[\prod_{k=1}^{n-1} \mathbb{P}\left\{F_{k} \cap F_{n}=\emptyset \mid M_{n}\right\}\right] \\
& =\mathbb{E}\left[\left(\mathbb{P}\left\{F_{1} \cap F_{2}=\emptyset \mid M_{2}\right\}\right)^{n-1}\right] .
\end{aligned}
$$

It therefore suffices by Borel-Cantelli to show that

$$
\begin{aligned}
\mathbb{E}\left[\sum_{n=1}^{\infty}\left(\mathbb{P}\left\{F_{1} \cap F_{2}=\emptyset \mid M_{2}\right\}\right)^{n-1}\right] & =\mathbb{E}\left[\frac{1}{1-\mathbb{P}\left\{F_{1} \cap F_{2}=\emptyset \mid M_{2}\right\}}\right] \\
& =\mathbb{E}\left[\frac{1}{\mathbb{P}\left\{F_{1} \cap F_{2} \neq \emptyset \mid M_{2}\right\}}\right] \\
& <\infty .
\end{aligned}
$$


Let $\nu$ be a fixed probability measure and put $S=\operatorname{supp} \nu$. Let $B(\epsilon), \epsilon>0$, be the $\epsilon$ ball around the identity in some metric that generates the topology on $\mathbb{G}$. Put $\beta_{\epsilon}=\lambda(B(\epsilon))^{-1}$. By a simple consequence of the Cauchy-Schwarz inequality (see Inequality II in Ch. 1 of [9]) we have

$$
\begin{aligned}
\mathbb{P}\left\{F_{1} \cap S \neq \emptyset\right\} & =\lim _{\epsilon \downarrow 0} \mathbb{P}\left\{\iint \mathbf{1}_{B(\epsilon)}\left(y z^{-1}\right) M_{1}(d y) \nu(d z)>0\right\} \\
& \geq \limsup _{\epsilon \downarrow 0} \frac{\left(\mathbb{E}\left[\beta_{\epsilon} M_{1} * \tilde{\nu}(B(\epsilon))\right]\right)^{2}}{\mathbb{E}\left[\left(\beta_{\epsilon} M_{1} * \tilde{\nu}(B(\epsilon))\right)^{2}\right]} \\
& =\left(\liminf _{\epsilon \downarrow 0} \mathbb{E}\left[\left(\beta_{\epsilon} M_{1} * \tilde{\nu}(B(\epsilon))\right)^{2}\right]\right)^{-1}
\end{aligned}
$$

(note that $\mathbb{E}\left[M_{1} * \tilde{\nu}(\cdot)\right]=\lambda$ by the assumption that $\sigma_{x} M_{1}$ has the same law as $M_{1}$ for all $x \in \mathbb{G}$ and the uniqueness of Haar measure).

Thus, by Fatou's lemma and Jensen's inequality we have (writing $\Lambda$ for the density of $M_{1} * \tilde{M}_{2}$ )

$$
\begin{aligned}
\mathbb{E}[ & \left.\frac{1}{\mathbb{P}\left\{F_{1} \cap F_{2} \neq \emptyset \mid M_{2}\right\}}\right] \\
& \leq \mathbb{E}\left[\liminf _{\epsilon \downarrow 0} \mathbb{E}\left[\left(\beta_{\epsilon} M_{1} * \tilde{M}_{2}(B(\epsilon))^{2} \mid M_{2}\right]\right]\right. \\
& \leq \liminf _{\epsilon \downarrow 0} \mathbb{E}\left[\left(\beta_{\epsilon} M_{1} * \tilde{M}_{2}(B(\epsilon))\right)^{2}\right] \\
& =\liminf _{\epsilon \downarrow 0} \mathbb{E}\left[\left(\beta_{\epsilon} \int \mathbf{1}_{B(\epsilon)}(y) \Lambda(y) \lambda(d y)\right)^{2}\right] \\
& =\liminf _{\epsilon \downarrow 0} \mathbb{E}\left[\int\left(\beta_{\epsilon} \int \mathbf{1}_{B(\epsilon)}(x y) \Lambda(y) \lambda(d y)\right)^{2} \lambda(d x)\right] \\
& \leq \liminf _{\epsilon \downarrow 0}\left[\int \beta_{\epsilon} \int \mathbf{1}_{B(\epsilon)}(x y) \Lambda(y)^{2} \lambda(d y) \lambda(d x)\right] \\
& \leq \liminf _{\epsilon \downarrow 0}\left[\beta_{\epsilon} \int\left(\int \mathbf{1}_{B(\epsilon)}(x y) \lambda(d x)\right)^{2} \Lambda(y)^{2} \lambda(d y)\right] \\
& =\mathbb{E}\left[\int \Lambda(y)^{2} \lambda(d y)\right] \\
& <\infty,
\end{aligned}
$$

and (6) holds as required.

\section{Proof of Theorem 1.1}

We need only show that (a) implies (b). Let $\Psi$ denote the characteristic exponent of $Y^{n}$; that is, $\mathbb{E}\left[\exp \left(i z \cdot Y_{t}^{n}\right)\right]=\exp (-t \Psi(z))$ for $t \geq 0, z \in \mathbb{Z}^{d}$. Write $v$ for the density of $\int_{0}^{\infty} e^{-t} \mathbb{P}\left\{Y_{t}^{n} \in \cdot\right\} d t$ with respect to $\ell$. By the same argument as in the proof of Theorem 5.4 of [7] we have $\int_{\mathbb{T}^{d}} v(x)^{2} \ell(d x)<\infty$. Note that $v$ has Fourier transform $(1+\Psi)^{-1}$ and so, by Parseval's theorem,

$$
\sum_{z \in \mathbb{Z}^{d}}\left|(1+\Psi(z))^{-1}\right|^{2}<\infty .
$$


Let $\check{Y}^{n}$ be the process $Y^{n}$ killed at an independent mean 1 exponential time. Put $Q_{n}=$ $\int_{0}^{\infty} \mathbf{1}\left\{\check{Y}_{t}^{n} \in \cdot\right\} d t$ and write $\hat{Q}_{n}$ for the Fourier transform of $Q_{n}$. It is easy to check that

$$
\mathbb{E}\left[\left|\hat{Q}_{n}(z)\right|^{2}\right]=2 \Re\left((1+\Psi(z))^{-1}\right)
$$

(see, for example, the proof of Theorem 2.2 in [4]). Therefore, by (7) and Parseval's theorem the random finite measure $Q_{i} * \tilde{Q}_{j}$ is absolutely continuous with respect to $\ell$ for $i \neq j$ with a density that is in $L^{2}(\ell \otimes \mathbb{P})$. Now put $M_{n}=T^{-1} \int_{0}^{T} \mathbf{1}\left\{X_{t}^{i} \in \cdot\right\} d t$. It is straightforward to conclude that $M_{i} * \tilde{M}_{j}$ is absolutely continuous with respect to $\ell$ for $i \neq j$ with a density that is in $L^{2}(\ell \otimes \mathbb{P})$.

An application of Proposition 3.1 completes the proof once it is noted that $\operatorname{supp} M_{n}$ is the closure of $\left\{X_{t}^{n}: 0 \leq t \leq T\right\}$ and that $\left\{X_{t}^{n}: 0 \leq t \leq T\right\}$ differs from its closure by at most a countable set.

\section{Proof of Theorem 1.2}

We need only show that (a) implies (b). We begin with some observations. Write $\bar{Y}_{t}=Y_{t}^{1}-Y_{t}^{2}$, so that $\bar{Y}$ is a symmetric Lévy process with characteristic exponent $2 \Re \Psi$, where $\Psi$, as above, is the characteristic exponent of $Y^{n}$. Statement (a) just says that points are not essentially polar for $\bar{Y}$, and by Kesten's condition (see, for example, Theorem II.16 of [3]) this is equivalent to $d=1$ and

$$
\sum_{z \in \mathbb{Z}} \frac{1}{1+2 \Re \Psi(z)}<\infty
$$

(the result in $[3]$ is stated for $\mathbb{R}^{d}$, but the same argument holds for $\mathbb{T}^{d}$ ).

By the same argument as in Theorem V.1 of [3], we see that if (8) holds, then for every $t>0$ the occupation measure $\int_{0}^{t} \mathbf{1}\left\{\bar{Y}_{s} \in \cdot\right\} d s$ is absolutely continuous with respect to $\ell$ with a density $L_{t}$ that is in $L^{2}(\ell \otimes \mathbb{P})$.

Put $G_{n}=\left\{\left(t, X_{t}^{n}\right): 0 \leq t \leq T\right\}$. We want to show that $\mathbb{P}\left(A_{n}\right.$ i.o. $)=0$, where $A_{n}$ is the event that $G_{n}$ does not intersect $\bigcup_{k=1}^{n-1} G_{k}$. Arguing as in the initial part of the proof of Proposition 3.1 that leads up to (6), it is sufficient to establish

$$
\mathbb{E}\left[\frac{1}{\mathbb{P}\left\{G_{1} \cap G_{2} \neq \emptyset \mid X^{2}\right\}}\right]<\infty .
$$

Observe by the quasi-left-continuity of $X^{1}$ and $X^{2}$ that

$$
\left\{\exists 0 \leq t \leq T: X_{t}^{1}=X_{t}^{2}\right\}=\left\{\sup _{\epsilon>0} \gamma_{\epsilon}\left(X^{1}, X^{2}\right) \leq T\right\} \text { a.s. }
$$

where for two càdlàg paths $h^{1}, h^{2}$ we set

$$
\gamma_{\epsilon}\left(h^{1}, h^{2}\right)=\inf \left\{t \geq 0:\left|h^{1}(t)-h^{2}(t)\right| \leq \epsilon\right\}
$$


(with the usual convention that inf $\emptyset=\infty$ ). Let $h$ be a fixed càdlàg path. By Cauchy-Schwarz we have

$$
\begin{aligned}
& \mathbb{P}\left\{\sup _{\epsilon \geq 0} \gamma_{\epsilon}\left(X^{1}, h\right) \leq T\right\}=\lim _{\epsilon \downarrow 0} \mathbb{P}\left\{\exists 0 \leq t \leq T:\left|X_{t}^{1}-h(t)\right| \leq \epsilon\right\} \\
& \geq \underset{\epsilon \downarrow 0}{\limsup } \mathbb{P}\left\{\int_{0}^{T} \mathbf{1}\left\{\left|X_{t}^{1}-h(t)\right| \leq \epsilon\right\} d t>0\right\} \\
& \geq \limsup _{\epsilon \downarrow 0} \frac{\left(\mathbb{E}\left[(\epsilon T / \pi)^{-1} \int_{0}^{T} \mathbf{1}\left\{\left|X_{t}^{1}-h(t)\right| \leq \epsilon\right\} d t\right]\right)^{2}}{\mathbb{E}\left[\left((\epsilon T / \pi)^{-1} \int_{0}^{T} \mathbf{1}\left\{\left|X_{t}^{1}-h(t)\right| \leq \epsilon\right\} d t\right)^{2}\right]} \\
& =\left(\liminf _{\epsilon \downarrow 0} \mathbb{E}\left[\left((\epsilon T / \pi)^{-1} \int_{0}^{T} \mathbf{1}\left\{\left|X_{t}^{1}-h(t)\right| \leq \epsilon\right\} d t\right)^{2}\right]\right)^{-1} .
\end{aligned}
$$

Therefore, by Fatou's lemma and Jensen's inequality,

$$
\begin{aligned}
\mathbb{E} & {\left[\frac{1}{\mathbb{P}\left\{G_{1} \cap G_{2} \neq \emptyset \mid X^{2}\right\}}\right] } \\
& \leq \mathbb{E}\left[\liminf _{\epsilon \downarrow 0} \mathbb{E}\left[\left((\epsilon T / \pi)^{-1} \int_{0}^{T} \mathbf{1}\left\{\left|X_{t}^{1}-X_{t}^{2}\right| \leq \epsilon\right\} d t\right)^{2} \mid X^{2}\right]\right] \\
& \leq \liminf _{\epsilon \downarrow 0} \mathbb{E}\left[\left((\epsilon T / \pi)^{-1} \int_{0}^{T} \mathbf{1}\left\{\left|X_{t}^{1}-X_{t}^{2}\right| \leq \epsilon\right\} d t\right)^{2}\right] \\
& =\liminf _{\epsilon \downarrow 0}\left[\int_{\mathbb{T}}\left((\epsilon T / \pi)^{-1} \int_{0}^{T} \mathbf{1}\left\{\left|x+\bar{Y}_{t}\right| \leq \epsilon\right\} d t\right)^{2} \ell(d x)\right] \\
& =\liminf _{\epsilon \downarrow 0}\left[T^{-2} \int_{\mathbb{T}}\left(\ell([x-\epsilon, x+\epsilon])^{-1} \int_{[x-\epsilon, x+\epsilon]} L_{T}(y) \ell(d y)\right)^{2} \ell(d x)\right] \\
& \leq \mathbb{E}\left[T^{-2} \int_{\mathbb{T}} L_{T}(x)^{2} \ell(d x)\right] \\
& <\infty,
\end{aligned}
$$

and (9) holds as required.

\section{CoAlescing LÉvy PRocesses: A PROBlem}

Our interest in the questions considered in this paper was sparked by a related, but apparently more difficult, problem concerning coalescing Lévy processes on the circle that arises in the analysis of the continuous sites stepping-stone models discussed in [6] and [5]. It would take us too far afield to describe these models and their genetic interpretation. However, we can briefly sketch the resulting Lévy process question.

Let $\left(X^{n}\right)_{n=1}^{\infty}$ be as in the introduction. For $n=1,2, \ldots$ define a process $\left(I_{t}^{n}\right)_{t \geq 0}$ taking values in the collection of subsets of $\{1, \ldots, n\}$ and stopping times $T_{0}^{n} \leq T_{1}^{n} \leq \ldots$ as follows. Put $I_{0}^{n}=\{1, \ldots, n\}$ and $T_{0}^{n}=0$. Suppose that $T_{0}^{n}, \ldots, T_{k}^{n}$ and $I_{t}^{n}$ for $t \in\left[0, T_{k}^{n}\right]$ have already 
been defined. Put

$$
T_{k+1}^{n}=\inf \left\{t>T_{k}^{n}: X_{t}^{i}=X_{t}^{j} \text { for some } i, j \in I_{T_{k}^{n}}^{n}, i \neq j\right\} .
$$

Set

$$
H=\min \left\{i \in I_{T_{k}^{n}}^{n}: X_{T_{k+1}^{n}}^{i}=X_{T_{k+1}^{n}}^{j} \text { for some } j \in I_{T_{k}^{n}}^{n}, i \neq j\right\}
$$

and

$$
L=\left\{j \in I_{T_{k}^{n}}^{n}: X_{T_{k+1}^{n}}^{H}=X_{T_{k+1}^{n}}^{j}\right\}
$$

(for clarity of notation, we don't record the dependence of $H$ and $L$ on $k$ and $n$ ). Put

$$
I_{t}^{n}=I_{T_{k}^{n}}^{n}, T_{k}^{n} \leq t<T_{k+1}^{n}, \text { and } I_{T_{k+1}^{n}}^{n}=I_{T_{k}^{n}}^{n} \backslash(L \backslash\{H\}) .
$$

The process $Z^{n}=\left(\left\{X_{t}^{i}: i \in I_{t}^{n}\right\}\right)_{t \geq 0}$, which takes values in the collection of finite subsets of $\mathbb{T}^{d}$ with $n$ or fewer points, is the usual system of $n$ coalescing Lévy processes: we have particles that move as independent copies of some Lévy process, except that when particles collide they are merged into a single particle.

Note that $I_{t}^{n}=I_{t}^{n+1} \cap\{1, \ldots, n\}$ for all $n$ and so $Z_{t}^{1} \subseteq Z_{t}^{2} \subseteq \ldots$ Put $Z_{t}^{\infty}=\bigcup_{n} Z_{t}^{n}$. The question arising from [6] and [5] is the following: Does the condition in part (a) of Theorem 1.2 imply that $Z_{t}^{\infty}$ is almost surely finite for all $t>0$ ? That is, if the Lévy particles can collide, then is it the case that the coalescing system starting with infinitely many particles instantaneously collapses down to only finitely many particles? It is clear that if this was the case, then part (b) of Theorem 1.2 would certainly hold, but the converse is not true a priori. The case when the underlying Lévy process is Brownian motion on $\mathbb{T}$ can be answered in the affirmative using the ideas in [1] or [2] (see, also, [8]). Some further remarks on the general case and a somewhat different approach to the Brownian case will appear in forthcoming joint work of the first author with Klaus Fleischmann, Tom Kurtz and Xiaowen Zhou.

Acknowledgment We thank the referee for useful comments concerning the counterexample in Section 2.

\section{REFERENCES}

[1] R. Arratia, Coalescing Brownian motions on the line, Ph.D. thesis, University of Wisconsin, 1979.

$[2] \longrightarrow$ Coalescing Brownian motions on $\mathbb{R}$ and the voter model on $\mathbb{Z}$, Preprint, 1981.

[3] J. Bertoin, Lévy Processes, Cambridge University Press, Cambridge, 1996.

[4] S.N. Evans, Multiple points in the sample paths of a Lévy process, Probab. Th. Rel. Fields 76 (1987), 359-367.

[5] C Coalescing Markov labelled partitions and a continuous sites genetics model with infinitely many types, Ann. Inst. Henri Poincaré B 33 (1997), 339-358.

[6] S.N. Evans and K. Fleischmann, Cluster formation in a stepping-stone model with continuous, hierarchically structured sites, Ann. Probab. 24 (1996), 1926-1952.

[7] P.J. Fitzsimmons and T.S. Salisbury, Capacity and energy for multiparameter Markov processes, Ann. Inst. Henri Poincaré 25 (1989), 325-350.

[8] T.E. Harris, Coalescing and noncoalescing stochastic flows in $\mathbb{R}_{1}$, Stochastic Process. Appl. 17 (1984), $187-210$.

[9] J.-P. Kahane, Some random series of functions, Cambridge University Press, Cambridge, 1985. 